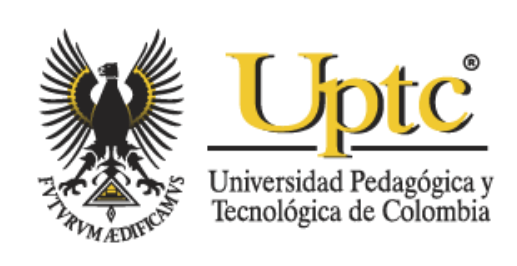

\title{
Uso de organofosforados por agricultores de la comunidad de Guaslán- Ecuador y los cambios hematológicos
}

Johanna Elizabeth Esparza-Olalla, Francy Carolina ForeroLugo \& Marcela Alejandra Mardones-Montanares

Citación: Esparza-Olalla, J. E., Forero-Lugo, F. C., \& Mardones-Montanares, M. A. (2020). Uso de organofosforados por agricultores de la comunidad de Guaslán- Ecuador y los cambios hematológicos. Ciencia y Agricultura, 17(1), 31-50.

https://doi.org/10.19053/01228420.v17.n1.2020.10603

Recibido: Septiembre 16, 2019; Aceptado: Diciembre 1, 2019; Publicado: Enero 15, 2020

Derechos de reproducción: Este es un artículo en acceso abierto distribuido bajo la licencia Creative Commons Attribution License (CC BY). 


\title{
Uso de organofosforados por agricultores de la comunidad de Guaslán- Ecuador y los cambios hematológicos
}

\author{
Johanna Elizabeth Esparza-Olalla ${ }^{1}$ \\ Francy Carolina Forero-Lugo ${ }^{2}$ \\ Marcela Alejandra Mardones-Montanares ${ }^{3}$
}

\section{Resumen}

En Ecuador el uso de plaguicidas organofosforados en la agricultura se ha convertido en un problema de salud pública, según datos bibliográficos las personas expuestas de manera ocupacional a estos agroquímicos manifiestan alteraciones en su salud. Este estudio fue enfocado en la valoración de parámetros hematológicos y morfológicos de agricultores expuestos de forma ocupacional a estos plaguicidas. Es un estudio observacional, descriptivo de corte transversal y correlacional. En la comunidad de Guaslán-Ecuador aproximadamente 400 personas se dedican a la agricultura, de los cuales 186 individuos formaron parte del estudio forma libre y voluntaria. El $53 \%$ de la población fueron mujeres, el 47\% hombres. La valoración del hemograma permitió determinar alteraciones cuantitativas como: poliglobulia, leucocitosis, neutrofilia, linfocitosis y cambios en las plaquetas. La morfología celular se valoró con el análisis del frotis de sangre periférica que evidenció alteraciones cualitativas en glóbulos rojos, blancos y plaquetas. Los hallazgos más relevantes fueron: neutrófilos con granulación tóxica, linfocitos reactivos, hipersegmentación, plaquetas grandes y estomatocitosis. La colinesterasa eritrocitaria obtuvo $13 \%$ de la población. El 99\% de la población presentó al menos una alteración cuantitativa y un $80 \%$ alteraciones cualitativas. La actividad de la colinesterasa resultó inversamente proporcional al tiempo de exposición. Se concluye que los plaguicidas organofosforados son capaces de producir alteraciones a nivel celular y enzimático. Se recomienda incluir el hemograma y el frotis de sangre periférica como marcadores de hemotoxicidad en pruebas ocupacionales 0 de perfil epidemiológico para personas expuestas a este tipo de agroquímicos.

Palabras clave: agricultores; colinesterasa; hemograma; organofosforados; salud pública.

\footnotetext{
${ }^{1}$ Pontificia Universidad Católica del Ecuador (Quito, Ecuador). ORCID: 0000-0002-3425-0599.

2 Pontificia Universidad Católica del Ecuador (Quito, Ecuador). ORCID: 0000-0001-5376-9102.

${ }^{3}$ M. Sc. Pontificia Universidad Católica del Ecuador (Quito, Ecuador). mmardones382@puce.edu.ec. ORCID: 0000-0002-5629-0567.
} 
Uso de organofosforados por agricultores de la comunidad de Guaslán- Ecuador y los cambios hematológicos

\title{
Use of organophosphates by farmers in the community of Guaslán- Ecuador and hematological changes
}

\begin{abstract}
In Ecuador, the use of organophosphorus pesticides in agriculture has become a public health problem, according to bibliographic data, people exposed to these agrochemicals in occupational ways show alterations in their health. This study was focused on the assessment of hematological and morphological parameters of farmers exposed in an occupational way to these pesticides. It is an observational, descriptive cross-sectional and correlational study. In the community of GuaslánEcuador approximately 400 people are engaged in agriculture, of which 186 individuals were part of the study freely and voluntarily. $53 \%$ of the population were women, $47 \%$ men. The assessment of the blood count allowed to determine quantitative alterations such as: polyglobulia, leukocytosis, neutrophilia, lymphocytosis and platelet changes. Cellular morphology was assessed with the analysis of the peripheral blood smear that showed qualitative alterations in red, white and platelet cells. The most relevant findings were: neutrophils with toxic granulation, reactive lymphocytes, hypersegmentation, large platelets and stomatocytosis. Erythrocyte cholinesterase obtained $13 \%$ of the population. The $99 \%$ of the population presented at least one quantitative alteration and $80 \%$ qualitative alterations. Cholinesterase activity was inversely proportional to the exposure time. It is concluded that organophosphorus pesticides are capable of producing alterations at the cellular and enzymatic level. It is recommended to include the blood count and peripheral blood smear as markers of hemotoxicity in occupational tests or epidemiological profile for people exposed to this type of agrochemicals.
\end{abstract}

Keywords: blood count; cholinesterase; farmers; organophosphorus; public health.

Para citar este artículo:

Esparza-Olalla, J. E., Forero-Lugo, F. C., \& Mardones-Montanares, M. A. (2020). Uso de organofosforados por agricultores de la comunidad de Guaslán- Ecuador y los cambios hematológicos. Ciencia $\quad y \quad$ Agricultura, $17(1), \quad 31-50$. https://doi.org/10.19053/01228420.v17.n1.2020.10603

Esta obra está bajo licencia internacional Creative Commons Reconocimiento 4.0

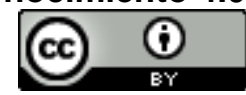




\section{INTRODUCCIÓN}

Los plaguicidas a nivel agrícola son una herramienta útil y aplicada que permite eliminar plagas que invaden los cultivos, esto mejora su producción y comercialización. Sin embargo, el uso indiscriminado y sin regulación de los agroquímicos hace que las plagas se vuelvan resistentes, por lo tanto se utilizan químicos más concentrados en su componente activo, altamente tóxicos sin considerar el efecto dañino para el agricultor y el medio ambiente (Mora Ortiz, 2014).

Los individuos expuestos de manera ocupacional al uso y manejo de organofosforados muchas veces manifiestan enfermedades graves, de aparición crónica con sintomatología que va desde irritación de piel, mucosas, cefalea, convulsiones, debilidad, problemas neurológicos, ansiedad, mareos, náuseas, vómitos, problemas de sueño y anorexia (Toro B. Rojas A. Díaz J., 2017).

El cuadro característico de intoxicación por plaguicidas organofosforados, es más conocido como síndrome colinérgico, debido a la estimulación exacerbada de los receptores de acetilcolina, como consecuencia de ello las manifestaciones clínicas más comunes son debilidad muscular, excesiva actividad secretora de la enzima acetilcolinesterasa y alteración del estado de conciencia (Fernández, Mancipe, \& Fernández, 2010). Algunos estudios realizados sobre plaguicidas organofosforados indican que estos agroquímicos generan la producción de radicales libres en el organismo, los cuales ocasionan lesiones a nivel de las membranas celulares ricas en lípidos mediante el mecanismo de lipoperoxidación. Este mecanismo provoca variaciones en las diferentes líneas celulares presentando alteraciones cualitativas y cuantitativas de las mismas (Cortez, 2017).

En Ecuador, las investigaciones sobre el efecto de plaguicidas organofosforados en la población se han enfocado al estudio del biomarcador recomendado por la Organización Mundial Salud (OMS) como indicador biológico de exposición, es decir medir la actividad de la colinesterasa; una disminución de la actividad superior al $30 \%$ sugiere supervisar y evaluar el estado de salud de la persona expuesta junto con un retiro pasajero del trabajo, mientras que la actividad inhibida de la enzima en más del $50 \%$ indica una intoxicación de tipo agudo teniendo en cuenta los síntomas clínicos en aquellos sujetos que no han tenido alteraciones anteriores (Fernández et al., 2010; Cortez, 2017).

La superficie agrícola ecuatoriana es de 2.813.217 hectáreas (ha) con cultivos permanentes, transitorios y de flores. De esta área, 1.320.988,67 ha utiliza algún tipo de plaguicida de origen químico en los sembríos, lo que representa al $47 \%$ de toda la superficie. De acuerdo con las estadísticas, 1 de cada 10 ha de los cultivos utilizan plaguicidas de categoría I, conocidos como extremadamente tóxicos. Teniendo en cuenta este dato es de suma importancia abordar temas relacionados con el efecto y las consecuencias que tienen los plaguicidas sobre el ser humano (INEC, 2013; INEC, 2016). 
Uso de organofosforados por agricultores de la comunidad de Guaslán- Ecuador y los cambios hematológicos

Varios aspectos como: la manera de fumigación, escasa información, ausencia de control, exceso en la dosis recomendada, tipo de almacenamiento y desecho final entre otros son preocupantes, que deben ser considerados para generar cambios favorables a los agricultores, consumidores y el medio ambiente, siendo este último de gran importancia, ya que Ecuador es el primer país a nivel mundial en reconocer a la naturaleza como sujeto de derecho, garantizando de esta manera la biodiversidad y los ecosistemas agrarios para generar soberanía alimentaria (Naranjo, 2017).

\section{Metodología}

La comunidad de Guaslán tiene un clima templado subandino, con una temperatura máxima de $18^{\circ}$ c y una mínima de $10^{\circ} \mathrm{c}$, humedad relativa del $75-80 \%$ La ubicación geográfica de la parroquia de San Luis es al Oeste del cantón Riobamba, provincia de Chimborazo con una altitud de $2662 \mathrm{msnm}$ (Santillán et al., 2014), formada por nueve comunidades, la cabecera parroquial y distribuida de la siguiente forma:

Tabla 1. Población según división territorial interna (GAD, 2014).

\begin{tabular}{|c|c|c|}
\hline Asentamiento Humano & Población & Superficie (Hectáreas) \\
\hline Cabecera parroquial & 1835 & 659,22 \\
\hline Candelaria & 615 & 317,45 \\
\hline Corazón de Jesús & 840 & 117,12 \\
\hline El Troje & 695 & 295,64 \\
\hline Guaslán & 1310 & 469,98 \\
\hline La Libertad & 2451 & 177,16 \\
\hline La Inmaculada & 2100 & 462,79 \\
\hline Monjas Tunshi & 300 & 139,48 \\
\hline San Antonio & 1130 & 169,73 \\
\hline San Vicente Tiazo & 780 & 118,35 \\
\hline
\end{tabular}

El estudio fue de tipo observacional, descriptivo, de corte transversal y correlacional. Se relacionaron las variables como las alteraciones de los contajes celulares con las variables sociodemográficas, tiempos de exposición a organofosforados, tipo de actividad que realizan en el campo, uso de equipos de protección y capacitaciones, entre otras. El muestreo para la investigación se realizó mediante una técnica probabilística aleatoria simple, en la cual todos los individuos de la población tuvieron la misma posibilidad de ser seleccionados.

Los participantes llenaron el consentimiento informado libre y voluntariamente y las muestras de sangre fueron extraídas según lo indica las normas del Comité Internacional de Estandarización de Laboratorios Clínicos (CLSI). El análisis de laboratorio se realizó con la ayuda de dos equipos automatizados: Mindray BC5800 para el hemograma y uso de reactivos comerciales (M-53P PROBE CLEANSER de $50 \mathrm{ml}$, M-58 LEO (I) LYSE de 1 litro, M-58 LH LYSE de $500 \mathrm{ml}, \mathrm{M}-$ 58 LEO (II) LYSE de 200ml, M-58 LBA LYSE de 1 litro y M-58D DILUENT de 20 litros, además se utilizó colorante Wright para la tinción de los frotis sanguíneos 
para la observación microscópica. Otro equipo utilizado fue Vital Scientific - Elitech Selectra XL-PRO para la prueba enzimática.

Una vez procesadas las muestras fueron eliminadas según en el acuerdo ministerial ํ5186 de los Ministerios de Salud Pública y Ambiente

Se creó una base de datos que fue importado al programa estadístico SPSS vs.24 para la codificación de las variables y la realización del análisis estadístico, resumido en tablas y gráficos que sirvieron para evidenciar la presencia de alteraciones cualitativas y/o cuantitativas en las células sanguíneas y la actividad de la colinesterasa eritrocitaria en los agricultores expuestos ocupacionalmente a plaguicidas organofosforados. Se utilizó la prueba de Ji cuadrado con un nivel de significancia estadística del 0,05 para establecer si existió o no relación entre las variables de estudio.

\section{Resultados}

En el grupo de agricultores que conformaron la muestra del estudio se encontró mayor participación de mujeres con un 53\% (98) frente al 47\% (88) para el grupo de los hombres.

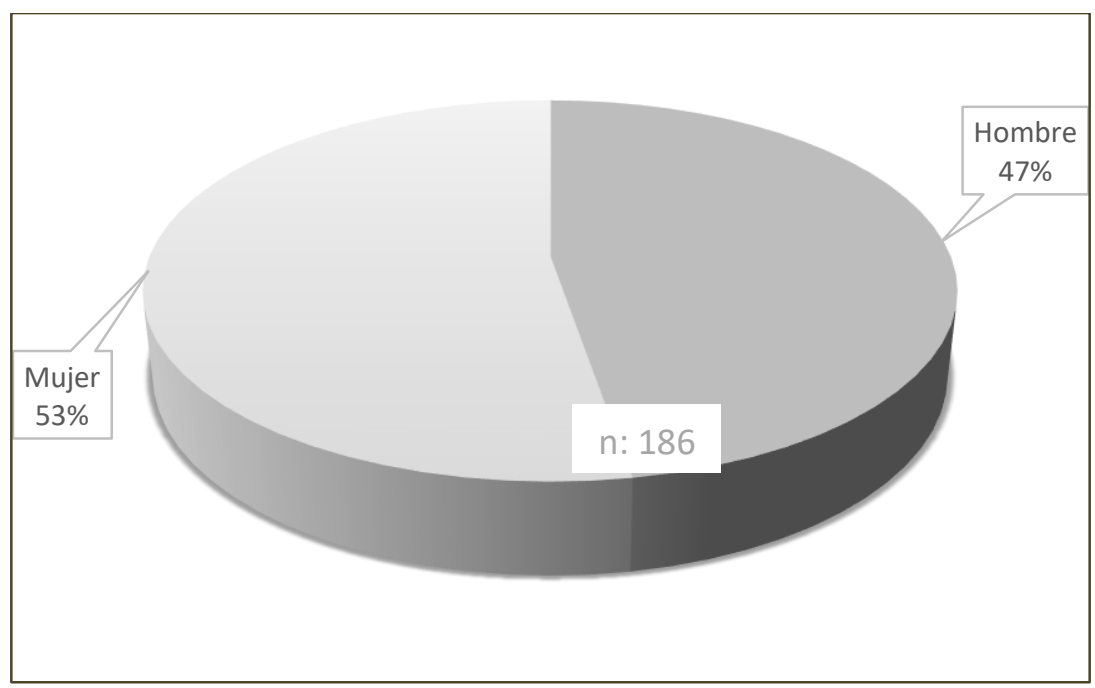

Fig. 1. Distribución de los agricultores por género.

De acuerdo a la edad el porcentaje mayor de agricultores se presenta de 29 a 49 años de edad (53\%).

Tabla 2. Distribución por edad.

\begin{tabular}{crr}
\hline Edad & Frecuencia & Porcentaje \\
\hline 18 a 28 años & 43 & $23 \%$ \\
29 a 49 años & 98 & $53 \%$ \\
> 49 años & 45 & $24 \%$ \\
Total & 186 & $100 \%$ \\
\hline
\end{tabular}


Uso de organofosforados por agricultores de la comunidad de Guaslán- Ecuador y los cambios hematológicos

El $41 \%$ de agricultores refirieron tener una superficie de cultivos menor a $2000 \mathrm{~m}^{2}$, el $31 \%$ sostuvo que la superficie de sus cultivos estaba dentro del rango de 2000 a $4000 \mathrm{~m}^{2}$ y por último un $28 \%$ indicó tener una superficie cultivada mayor a 4000 $\mathrm{m}^{2}$ dedicados al cultivo de hortalizas que corresponde al $58 \%$ y el $7 \%$ al cultivo de frutas. Un grupo importante de agricultores se dedican al cultivo mixto, es decir tanto frutas como hortalizas, $35 \%$.

Un $70 \%$ de los agricultores aplican en sus cultivos entre 100 a 200 litros de solución con plaguicida durante la fumigación. Un 14\% y un $16 \%$ usan volúmenes menores a 100 litros y mayores a 200 respectivamente. Estas fumigaciones las realizan los agricultores cada quince días (46\%), seguido por aquellos que las realizan una vez por semana, el $28 \%(n=52)$, porcentaje no despreciable dado el impacto que tienen los plaguicidas en la comunidad en general y por último 49 de 186 agricultores fumigan los cultivos una vez al mes, que representa el $26 \%$.

Con la finalidad de relacionar el tiempo de exposición a plaguicidas organofosforados y posibles alteraciones en las células sanguíneas, se preguntó a los agricultores sobre el tiempo al que llevan expuestos a plaguicidas organofosforados, el mayor porcentaje fue de $51 \%$ de los agricultores expuestos de 15 a 30 años.

Tabla 3. Superficie cultivada, cultivos y prácticas de fumigación.

\begin{tabular}{cccc}
\hline Variable & Categoría & $\mathbf{n}$ & $\%$ \\
\hline Superficie cultivada & $<2000$ & 76 & 40,9 \\
$\left(\mathrm{~m}^{2}\right)$ & 2000 a 4000 & 57 & 30,6 \\
& $>4000$ & 53 & 28,5 \\
\hline \multirow{2}{*}{ Tipo de cultivo } & Hortalizas & 107 & 57,5 \\
& Frutas y hortalizas & 66 & 35,5 \\
& Frutas & 13 & 7,0 \\
\hline \multirow{2}{*}{ Frecuencia de } & Cada 15 días & 85 & 45,7 \\
fumigación & Una vez por semana & 52 & 28,0 \\
& Una vez al mes & 49 & 26,3 \\
\hline \multirow{2}{*}{ Volumen de solución } & 100 a 200 litros & 130 & 69,9 \\
de plaguicida & $>200$ litros & 30 & 16,1 \\
& $<100$ litros & 26 & 14,0 \\
\hline Tiempo de & 15 a 30 años & 95 & 51,1 \\
exposición del & $<15$ años & 48 & 25,8 \\
agricultor & $>30$ años & 43 & 23,1 \\
\hline Uso de EPP£ & No usa & 116 & 64,4 \\
& Usa & 70 & 37,6 \\
\hline Equipo de Protección Personal. & Total & 186 & 100,0 \\
\hline
\end{tabular}

De 186 participantes, 70 indicaron que utilizan equipos de protección personal mientras que 116 admitieron no utilizar EPP, sin embargo, se constató que estos 
no son los equipos adecuados para la actividad que realizan, según lo recomendado por organismos internacionales.

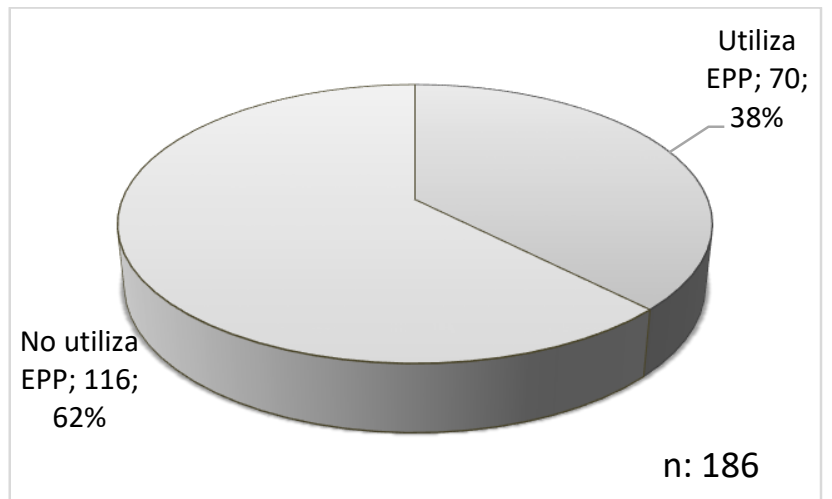

Fig. 2. Uso de equipo de protección personal para labores de campo.

De la relación de variables, se pudo establecer una asociación altamente significativa entre los niveles de hemoglobina y del hematocrito vs el género de los agricultores, mediante el análisis de la prueba de Ji cuadrado se obtuvo un $p=0,000$ y 0,001 para hemoglobina y hematocrito respectivamente.

Tabla 4. Niveles de hemoglobina, hematocrito y variables sociodemográficas; actividades de los agricultores y de fumigación.

\begin{tabular}{|c|c|c|c|c|c|c|c|c|c|c|c|c|c|}
\hline \multicolumn{4}{|c|}{ Parámetros } & \multicolumn{5}{|c|}{ Nivel de hemoglobina } & \multicolumn{5}{|c|}{ Nivel de hematocrito } \\
\hline \multirow[t]{2}{*}{$\begin{array}{l}\text { Varia } \\
\text { bles }\end{array}$} & \multirow[t]{2}{*}{$\begin{array}{l}\text { Categoría } \\
\mathbf{s}\end{array}$} & \multicolumn{2}{|c|}{ Total } & \multicolumn{2}{|c|}{$\begin{array}{c}\text { Normal } \\
\text { (H: } 14.90- \\
18.30 \mathrm{~g} / \mathrm{dL} ; \\
\text { M: } 12.70- \\
16.20 \mathrm{~g} / \mathrm{dL})\end{array}$} & \multicolumn{2}{|c|}{$\begin{array}{c}\text { Alto } \\
\text { (H: >18 } \\
\text { g/dL; } \\
\text { M: >16 } \\
\text { g/dL) }\end{array}$} & \multirow[t]{2}{*}{$\mathbf{p}$} & \multicolumn{2}{|c|}{$\begin{array}{c}\text { Normal } \\
\text { (H: } \\
43.30- \\
52.80 \% ; \\
\text { M: } 37.90- \\
47 \%)\end{array}$} & \multicolumn{2}{|c|}{$\begin{array}{c}\text { Alto } \\
\text { (H: >53\%; } \\
M:>47 \%)\end{array}$} & \multirow[t]{2}{*}{$\mathbf{p}$} \\
\hline & & $\mathbf{n}$ & $\%$ & $\mathbf{n}$ & $\%$ & $\mathbf{n}$ & $\%$ & & $\mathbf{n}$ & $\%$ & $\mathbf{n}$ & $\%$ & \\
\hline \multirow{2}{*}{\begin{tabular}{l}
$\bar{\Phi}$ \\
$\stackrel{\bar{\Phi}}{\Phi}$ \\
\hdashline
\end{tabular}} & Hombre & 88 & 47,3 & 20 & 10,8 & 68 & 36,6 & \multirow{2}{*}{$0,001^{* *}$} & 56 & 30,1 & 32 & 17,2 & \multirow{2}{*}{$0,000^{* *}$} \\
\hline & Mujer & 98 & 52,7 & 45 & 24,2 & 53 & 28,5 & & 33 & 17,7 & 65 & 34,9 & \\
\hline \multirow{3}{*}{ 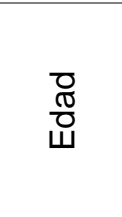 } & $\begin{array}{l}18 \text { a } 28 \\
\text { años }\end{array}$ & 43 & 23,1 & 12 & 6,5 & 31 & 16,7 & \multirow{3}{*}{0,247} & 20 & 10,8 & 23 & 12,4 & \multirow{3}{*}{0,800} \\
\hline & $\begin{array}{l}29 \text { a } 49 \\
\text { años }\end{array}$ & 98 & 52,7 & 33 & 17,7 & 65 & 34,9 & & 46 & 24,7 & 52 & 28,0 & \\
\hline & > 49 años & 45 & 24,2 & 20 & 10,8 & 25 & 13,4 & & 23 & 12,4 & 22 & 11,8 & \\
\hline \multirow{4}{*}{ 要 } & Fumiga & 9 & 4,8 & 1 & 0,5 & 8 & 4,3 & \multirow{4}{*}{0,328} & 7 & 3,8 & 2 & 1,1 & \multirow{4}{*}{0,281} \\
\hline & Siembra & 5 & 2,7 & 1 & 0,5 & 4 & 2,2 & & 3 & 1,6 & 2 & 1,1 & \\
\hline & Cosecha & 6 & 3,2 & 3 & 1,6 & 3 & 1,6 & & 3 & 1,6 & 3 & 1,6 & \\
\hline & Todas & 166 & 89,2 & 60 & 32,3 & 106 & 57,0 & & 76 & 40,9 & 90 & 48,4 & \\
\hline \multirow{3}{*}{ 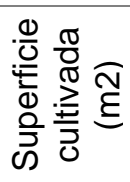 } & $<2000$ & 76 & 40,9 & 21 & 11,3 & 55 & 29,6 & \multirow{3}{*}{0,118} & 28 & 15,1 & 48 & 25,8 & \multirow{3}{*}{$0,009^{* *}$} \\
\hline & $\begin{array}{l}2000 \mathrm{a} \\
4000\end{array}$ & 57 & 30,6 & 20 & 10,8 & 37 & 19,9 & & 27 & 14,5 & 30 & 16,1 & \\
\hline & $>4000$ & 53 & 28,5 & 24 & 12,9 & 29 & 15,6 & & 34 & 18,3 & 19 & 10,2 & \\
\hline \multirow{2}{*}{ 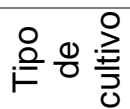 } & Frutas & 13 & 7,0 & 4 & 2,2 & 9 & 4,8 & \multirow{2}{*}{0,807} & 6 & 3,2 & 7 & 3,8 & \multirow{2}{*}{0,575} \\
\hline & Hortalizas & 107 & 57,5 & 36 & 19,4 & 71 & 38,2 & & 48 & 25,8 & 59 & 31,7 & \\
\hline
\end{tabular}


Uso de organofosforados por agricultores de la comunidad de Guaslán- Ecuador y los cambios hematológicos

\begin{tabular}{|c|c|c|c|c|c|c|c|c|c|c|c|c|c|}
\hline \multirow{2}{*}{\multicolumn{2}{|c|}{$\begin{array}{c}\text { Parámetros } \\
\begin{array}{c}\text { Frutas y } \\
\text { hortalizas }\end{array}\end{array}$}} & \multirow[b]{2}{*}{66} & \multirow[b]{2}{*}{35,5} & \multicolumn{5}{|c|}{ Nivel de hemoglobina } & \multicolumn{5}{|c|}{ Nivel de hematocrito } \\
\hline & & & & 25 & 13,4 & 41 & 22,0 & & 35 & 18,8 & 31 & 16,7 & \\
\hline \multirow{3}{*}{ 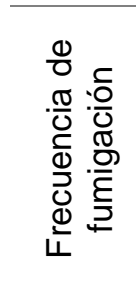 } & $\begin{array}{l}\text { Una vez } \\
\text { por } \\
\text { semana }\end{array}$ & 52 & 28,0 & 17 & 9,1 & 35 & 18,8 & \multirow{3}{*}{0,378} & 31 & 16,7 & 21 & 11,3 & \multirow{3}{*}{0,123} \\
\hline & $\begin{array}{l}\text { Cada } 15 \\
\text { días }\end{array}$ & 85 & 45,7 & 34 & 18,3 & 51 & 27,4 & & 38 & 20,4 & 47 & 25,3 & \\
\hline & $\begin{array}{l}\text { Una vez al } \\
\text { mes }\end{array}$ & 49 & 26,3 & 14 & 7,5 & 35 & 18,8 & & 20 & 10,8 & 29 & 15,6 & \\
\hline \multirow{3}{*}{ 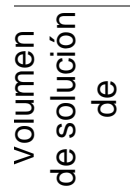 } & $\frac{1}{2}<100$ litros & 26 & 14,0 & 5 & 2,7 & 21 & 11,3 & \multirow{3}{*}{0,193} & 12 & 6,5 & 14 & 7,5 & \multirow{3}{*}{0,573} \\
\hline & $\begin{array}{l}100 \text { a } 200 \\
\text { litros }\end{array}$ & 130 & 69,9 & 49 & 26,3 & 81 & 43,5 & & 60 & 32,3 & 70 & 37,6 & \\
\hline & $>200$ litros & 30 & 16,1 & 11 & 5,9 & 19 & 10,2 & & 17 & 9,1 & 13 & 7,0 & \\
\hline \multirow{3}{*}{ 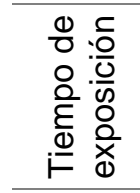 } & $<15$ años & 48 & 25,8 & 12 & 6,5 & 36 & 19,4 & \multirow{3}{*}{0,240} & 23 & 12,4 & 25 & 13,4 & \multirow{3}{*}{0,979} \\
\hline & $\begin{array}{l}15 \text { a } 30 \\
\text { años }\end{array}$ & 95 & 51,1 & 36 & 19,4 & 59 & 31,7 & & 46 & 24,7 & 49 & 26,3 & \\
\hline & > 30 años & 43 & 23,1 & 17 & 9,1 & 26 & 14,0 & & 20 & 10,8 & 23 & 12,4 & \\
\hline \multirow{3}{*}{$\stackrel{\mathscr{O}}{\supset} \frac{0}{0}$} & Usa & 70 & 37,6 & 17 & 9,1 & 53 & 28,5 & \multirow{2}{*}{0,118} & 31 & 16,7 & 39 & 21,0 & \multirow{2}{*}{0,450} \\
\hline & No usa & 116 & 62,4 & 48 & 25,8 & 68 & 36,6 & & 58 & 31,2 & 58 & 31,2 & \\
\hline & Total & 186 & 100 & 65 & 34.9 & 121 & 65.1 & & 89 & 47,8 & 97 & 52,2 & \\
\hline
\end{tabular}

En la tabla 5 el 9,6\% de los agricultores presentó valores altos en el contaje celular para glóbulos blancos, los porcentajes más altos se encontraron en los agricultores que poseen tierras con más de $4000 \mathrm{~m}^{2}$ de cultivo $(4,3 \%)$, cultivan hortalizas $(5,4 \%)$, fumigan cada semana y cada quince días $(7,6 \%)$, con un volumen de 100 a 200 litros $(6,5 \%)$ y los que están expuestos a productos químicos para fumigación entre 25 y 30 años $(4,8 \%)$.

Tabla 5. Nivel de glóbulos blancos y variables sociodemográficas, actividades de los agricultores y

\begin{tabular}{|c|c|c|c|c|c|c|c|c|}
\hline \multicolumn{2}{|c|}{$\begin{array}{c}\text { Nivel de glóbulos } \\
\text { blancos }\end{array}$} & \multicolumn{2}{|c|}{$\begin{array}{l}\text { Normal } \\
(4.0-10.0 \mathrm{~K} / \square \mathrm{l})\end{array}$} & \multicolumn{2}{|c|}{$\begin{array}{l}\text { Alto } \\
(>10.0 \\
K / \square I)\end{array}$} & \multicolumn{2}{|c|}{$\begin{array}{l}\text { Total en } \\
\text { muestra }\end{array}$} & \multirow[t]{2}{*}{$\mathbf{p}$} \\
\hline Variable & Categoría & $\mathbf{n}$ & $\%$ & $\mathbf{n}$ & $\%$ & $\mathbf{n}$ & $\%$ & \\
\hline \multirow{2}{*}{ 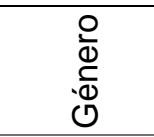 } & Hombre & 79 & 42,5 & 9 & 4,8 & 88 & 47,3 & \multirow{2}{*}{0,81} \\
\hline & Mujer & 89 & 47,8 & 9 & 4,8 & 98 & 52,7 & \\
\hline \multirow{3}{*}{ 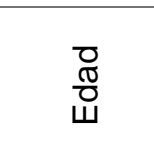 } & 18 a 28 años & 34 & 24,2 & 9 & 4,3 & 43 & 23,1 & \multirow{3}{*}{$0,015^{*}$} \\
\hline & 29 a 49 años & 91 & 5,9 & 7 & 1,1 & 98 & 52,7 & \\
\hline & > 49 años & 43 & 52,2 & 2 & 5,4 & 45 & 24,2 & \\
\hline \multirow{4}{*}{ 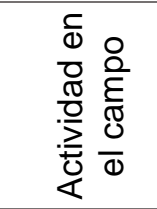 } & Fumiga & 7 & 3,8 & 2 & 1,1 & 9 & 4,8 & \multirow{4}{*}{ a } \\
\hline & Siembra & 4 & 2,2 & 1 & 0,5 & 5 & 2,7 & \\
\hline & Cosecha & 4 & 2,2 & 2 & 1,1 & 6 & 3,2 & \\
\hline & Todas & 153 & 82,3 & 13 & 7,0 & 166 & 89,2 & \\
\hline () $\overline{\overline{0}} \geqq \overline{\widetilde{\sigma}}$ & $<2000$ & 70 & 37,6 & 6 & 3,2 & 88 & 47,3 & 0,284 \\
\hline
\end{tabular}

Ciencia y Agricultura (Cien. Agri.) Vol. 17 (1). L-ISSN 0122-8420. elSSN 2539-0899.

Enero-Abril 2020, pp. 31-50. Tunja (Boyacá) - Colombia. DOI: https://doi.org/10.19053/01228420.v17.n1.2020.10603 


\begin{tabular}{|c|c|c|c|c|c|c|c|c|}
\hline \multicolumn{2}{|c|}{$\begin{array}{l}\text { Nivel de glóbulos } \\
\text { blancos }\end{array}$} & \multicolumn{2}{|c|}{$\begin{array}{l}\text { Normal } \\
(4.0-10.0 \mathrm{~K} / \square \mathrm{I})\end{array}$} & \multicolumn{2}{|c|}{$\begin{array}{r}\text { Alto } \\
(>10.0 \\
K / \square I)\end{array}$} & \multicolumn{2}{|c|}{$\begin{array}{l}\text { Total en } \\
\text { muestra }\end{array}$} & \multirow[t]{2}{*}{$\mathbf{p}$} \\
\hline Variable & Categoría & $\mathbf{n}$ & $\%$ & $\mathbf{n}$ & $\%$ & $\mathbf{n}$ & $\%$ & \\
\hline & 2000 a 4000 & 53 & 28,5 & 4 & 2,2 & 98 & 52,7 & \\
\hline & $>4000$ & 45 & 24,2 & 8 & 4,3 & 43 & 23,1 & \\
\hline \multirow{3}{*}{ 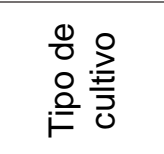 } & Frutas & 11 & 5,9 & 2 & 1,1 & 98 & 52,7 & \multirow{3}{*}{0,770} \\
\hline & Hortalizas & 97 & 52,2 & 10 & 5,4 & 45 & 24,2 & \\
\hline & Frutas y hortalizas & 60 & 32,3 & 6 & 3,2 & 9 & 4,8 & \\
\hline \multirow{3}{*}{ 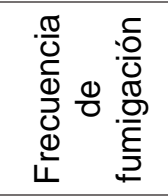 } & $\begin{array}{l}\text { Una vez por } \\
\text { semana }\end{array}$ & 45 & 24,2 & 7 & 3,8 & 5 & 2,7 & \multirow{3}{*}{0,554} \\
\hline & Cada 15 días & 78 & 41,9 & 7 & 3,8 & 6 & 3,2 & \\
\hline & Una vez al mes & 45 & 24,2 & 4 & 2,2 & 166 & 89,2 & \\
\hline \multirow{3}{*}{ 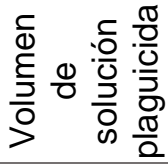 } & $<100 \mid$ & 24 & 12,9 & 2 & 1,1 & 26 & 14,0 & \multirow{3}{*}{0,739} \\
\hline & 100 a 200 I & 118 & 63,4 & 12 & 6,5 & 135 & 69,9 & \\
\hline & $>2001$ & 26 & 14,0 & 4 & 2,2 & 30 & 16,1 & \\
\hline \multirow{3}{*}{ 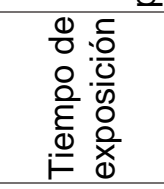 } & $<15$ años & 40 & 21,5 & 8 & 4,3 & 48 & 25,8 & \multirow{3}{*}{0,069} \\
\hline & 15 a 30 años & 86 & 46,2 & 9 & 4,8 & 95 & 51,1 & \\
\hline & > 30 años & 42 & 22,6 & 1 & 0,5 & 43 & 23,1 & \\
\hline \multirow{3}{*}{ 욤 움 } & Usa & 59 & 31,7 & 11 & 5,9 & 70 & 37,6 & \multirow{2}{*}{$0,031^{*}$} \\
\hline & No usa & 109 & 58,6 & 7 & 3,8 & 116 & 62,4 & \\
\hline & Total & 168 & 90.3 & 18 & 9.6 & 186 & 100 & \\
\hline
\end{tabular}

Nota: $p$-Test Ji cuadrado: *asociación estadísticamente significativa; EPP: Equipo de protección personal. a: no se realiza la prueba Ji cuadrado por el número de casos muy bajo o cero en las celdas.

En la tabla 6, el porcentaje mayor fue para neutropenia y para neutrofilia se encontró en las tres variables, así los que se dedican a todas las actividades en el campo presentaron el $25,3 \%$ de nivel bajo y el $3,2 \%$ de nivel alto; los que cultivan hortalizas con presencia de neutropenia el $18,3 \%$ y con neutrofilia el $3,8 \%$, finalmente aquellos que utilizan entre 100 y 200 litros de solución para fumigación, $21 \%$ con neutropenia y el $4,9 \%$ con neutrofilia.

Las relaciones nivel de neutrófilos (\%) y la superficie de tierra cultivada fue estadísticamente significativa (p. 0,050). Los datos recogidos para los linfocitos, eosinófilos, monocitos y basófilos no fue representativa para que sea evidenciado en tablas.

Tabla 6. Porcentaje de neutrófilos y variables sociodemográficas, actividades de los agricultores y de fumigación.

\begin{tabular}{|c|c|c|c|c|c|c|c|c|c|c|}
\hline \multicolumn{2}{|c|}{$\begin{array}{c}\text { Nivel de neutrófilos (fórmula } \\
\text { leucocitaria) }\end{array}$} & \multicolumn{2}{|c|}{$\begin{array}{c}\text { Bajo } \\
(<50 \%)\end{array}$} & \multicolumn{2}{|c|}{$\begin{array}{l}\text { Normal } \\
(50-70 \%)\end{array}$} & \multicolumn{2}{|c|}{$\begin{array}{l}\text { Alto } \\
(>70 \%)\end{array}$} & \multicolumn{2}{|c|}{ Total en muestra } & \multirow[t]{2}{*}{$\mathbf{p}$} \\
\hline Variable & Categoría & $\mathbf{n}$ & $\%$ & $\mathbf{n}$ & $\%$ & $\mathbf{n}$ & $\%$ & $\mathrm{n}$ & $\%$ & \\
\hline \multirow{2}{*}{ 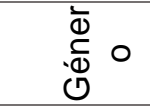 } & Hombre & 24 & 12,9 & 58 & 31,2 & 6 & 3,2 & 88 & 47,3 & \multirow{2}{*}{0,661} \\
\hline & Mujer & 30 & 16,1 & 64 & 34,4 & 4 & 2,2 & 98 & 52,7 & \\
\hline 무 & 18 a 28 años & 8 & 4,3 & 30 & 16,1 & 5 & 2,7 & 43 & 23,1 & 0,143 \\
\hline
\end{tabular}

Ciencia y Agricultura (Cien. Agri.) Vol. 17 (1). L-ISSN 0122-8420. elSSN 2539-0899.

Enero-Abril 2020, pp. 31-50. Tunja (Boyacá) - Colombia. DOI: https://doi.org/10.19053/01228420.v17.n1.2020.10603 
Uso de organofosforados por agricultores de la comunidad de Guaslán- Ecuador y los cambios hematológicos

\begin{tabular}{|c|c|c|c|c|c|c|c|c|c|c|}
\hline \multicolumn{2}{|c|}{$\begin{array}{c}\text { Nivel de neutrófilos (fórmula } \\
\text { leucocitaria) }\end{array}$} & \multicolumn{2}{|c|}{$\begin{array}{c}\text { Bajo } \\
(<50 \%)\end{array}$} & \multicolumn{2}{|c|}{$\begin{array}{l}\text { Normal } \\
(50-70 \%)\end{array}$} & \multicolumn{2}{|c|}{$\begin{array}{c}\text { Alto } \\
(>70 \%)\end{array}$} & \multicolumn{2}{|c|}{ Total en muestra } & \multirow[t]{2}{*}{$\mathbf{p}$} \\
\hline Variable & Categoría & $\mathbf{n}$ & $\%$ & $\mathbf{n}$ & $\%$ & $\mathbf{n}$ & $\%$ & $\mathbf{n}$ & $\%$ & \\
\hline & 29 a 49 años & 30 & 16,1 & 65 & 34,9 & 3 & 1,6 & 98 & 52,7 & \\
\hline & > 49 años & 16 & 8,6 & 27 & 14,5 & 2 & 1,1 & 45 & 24,2 & \\
\hline \multirow{4}{*}{ 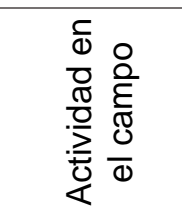 } & Fumiga & 2 & 1,1 & 5 & 2,7 & 2 & 1,1 & 9 & 4,8 & \\
\hline & Siembra & 3 & 1,6 & 2 & 1,1 & 0 & 0,0 & 5 & 2,7 & \\
\hline & Cosecha & 2 & 1,1 & 2 & 1,1 & 2 & 1,1 & 6 & 3,2 & a \\
\hline & Todas & 47 & 25,3 & 113 & 60,8 & 6 & 3,2 & 166 & 89,2 & \\
\hline \multirow{3}{*}{ 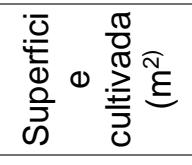 } & $<2000$ & 23 & 12,4 & 51 & 27,4 & 2 & 1,1 & 76 & 40,9 & \multirow{3}{*}{$0,050^{*}$} \\
\hline & 2000 a 4000 & 16 & 8,6 & 40 & 21,5 & 1 & 0,5 & 57 & 30,6 & \\
\hline & $>4000$ & 15 & 8,1 & 31 & 16,6 & 7 & 3,8 & 53 & 28,5 & \\
\hline \multirow{3}{*}{$\begin{array}{l}\frac{0}{0} \\
\text { 을 } \\
\text { 을 }\end{array}$} & Frutas & 3 & 1,6 & 10 & 5,4 & 0 & 0,0 & 13 & 7,0 & \multirow{3}{*}{0,661} \\
\hline & Hortalizas & 34 & 18,3 & 66 & 35,5 & 7 & 3,8 & 107 & 57,5 & \\
\hline & Frutas y hortalizas & 17 & 9,1 & 46 & 24,7 & 3 & 1,6 & 66 & 35,5 & \\
\hline \multirow{3}{*}{ 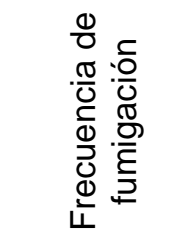 } & Una vez por semana & 13 & 7,0 & 34 & 18,2 & 5 & 2,7 & 52 & 28,0 & \multirow{3}{*}{0,586} \\
\hline & Cada 15 días & 26 & 14.0 & 56 & 30,1 & 3 & 1,6 & 85 & 45,7 & \\
\hline & Una vez al mes & 15 & 8,1 & 32 & 17,2 & 2 & 1,1 & 49 & 26,3 & \\
\hline \multirow{3}{*}{ 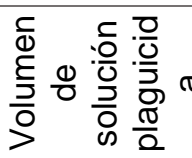 } & $<100 \mid$ & 7 & 3,8 & 19 & 10,2 & 0 & 0,0 & 26 & 14,0 & \multirow{3}{*}{0,594} \\
\hline & $: 100$ a $200 ।$ & 39 & 21.0 & 82 & 44,1 & 9 & 4,9 & 130 & 69,9 & \\
\hline & $>2001$ & 8 & 4,3 & 21 & 11,3 & 1 & 0,5 & 30 & 16,1 & \\
\hline \multirow{3}{*}{ 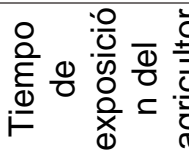 } & $<15$ años & 13 & 7,0 & 30 & 16,1 & 5 & 2,7 & 48 & 25,8 & \multirow{3}{*}{0,367} \\
\hline & 15 a 30 años & 26 & 14.0 & 66 & 35,5 & 3 & 1,6 & 95 & 51,1 & \\
\hline & $>30$ años & 15 & 8,1 & 26 & 14,0 & 2 & 1,1 & 43 & 23,1 & \\
\hline \multirow{3}{*}{ 옥 음 } & Usa & 22 & 11,8 & 43 & 23,1 & 5 & 2,7 & 70 & 37,6 & \multirow{2}{*}{0,557} \\
\hline & No usa & 32 & 17,2 & 79 & 42,5 & 5 & 2,7 & 116 & 62,4 & \\
\hline & Total & 54 & 29.0 & 122 & 65.6 & 10 & 5.4 & 186 & 100 & \\
\hline
\end{tabular}

Nota: $p$-Test Ji cuadrado: *asociación estadísticamente significativa; EPP: Equipo de protección personal. a: no se realiza la prueba Ji cuadrado por el número de casos muy bajo o cero en las celdas

En la Tabla 7 se detectó la presencia de estomatocitosis (morfología) característico en pacientes con afectación al hígado en un $2.7 \%$ en mujeres y en agricultores que no utilizan el EPP se observa en un $3.2 \%$, agricultores susceptibles de presentar una enfermedad 
Johanna Elizabeth Esparza-Olalla, Francy Carolina Forero-Lugo, Marcela Alejandra Mardones-

Montanares

Tabla 7. Morfología de glóbulos rojos y variables sociodemográficas, actividades de los agricultores y de fumigación.

\begin{tabular}{|c|c|c|c|c|c|c|c|c|c|c|}
\hline \multirow{2}{*}{$\begin{array}{c}\begin{array}{c}\text { Morfología } \\
\text { células } \\
\text { sanguíneas serie } \\
\text { roja a }^{\text {a }}\end{array} \\
\text { Variable/ } \\
\text { Categoría }\end{array}$} & \multirow{2}{*}{\multicolumn{2}{|c|}{$\begin{array}{r}\begin{array}{r}\text { Normocró } \\
\text { mico }\end{array} \\
\text { normocític } \\
0 \\
\text { n } \quad \%\end{array}$}} & \multicolumn{2}{|c|}{$\begin{array}{c}\text { Normocróm } \\
\text { ico } \\
\text { presencia } \\
\text { estomatocit } \\
\text { os }\end{array}$} & \multicolumn{2}{|c|}{$\begin{array}{c}\text { Hipercrómi } \\
\text { co, } \\
\text { normocític } \\
0\end{array}$} & \multicolumn{2}{|c|}{$\begin{array}{r}\text { Hipocrómi } \\
\text { co, } \\
\text { normocític } \\
0\end{array}$} & \multicolumn{2}{|c|}{$\begin{array}{l}\text { Total en } \\
\text { muestra }\end{array}$} \\
\hline & & & $\mathbf{n}$ & $\%$ & $\mathbf{n}$ & $\%$ & $\mathbf{n}$ & $\%$ & $\mathbf{n}$ & $\%$ \\
\hline$\stackrel{亠}{\Phi}$ Hombre & 76 & 40,9 & 3 & 1,6 & 8 & 4,3 & 1 & 0,5 & 88 & 47,3 \\
\hline Mujer & 92 & 49,5 & 5 & 2,7 & 1 & 0,5 & 0 & 0,0 & 98 & 52,7 \\
\hline $\begin{array}{l}18 \text { a } 28 \\
\text { años }\end{array}$ & 37 & 19,9 & 2 & 1,1 & 4 & 2,2 & 0 & 0,0 & 43 & 23,1 \\
\hline $\begin{array}{ll}\frac{\mathrm{d}}{0} & 29 \text { a } 49 \\
\text { ய } & \text { años }\end{array}$ & 89 & 47,8 & 4 & 2,2 & 4 & 2,2 & 1 & 0,5 & 98 & 52,7 \\
\hline > 49 años & 42 & 22,6 & 2 & 1,1 & 1 & 0,5 & 0 & 0,0 & 45 & 24,2 \\
\hline ฮิ & 6 & 3,2 & 2 & 1,1 & 1 & 0,5 & 0 & 0,0 & 9 & 4,8 \\
\hline 율 읕 Siembra & 4 & 2,2 & 1 & 0,5 & 0 & 0,0 & 0 & 0,0 & 5 & 2,7 \\
\hline$\sum$ O Cosecha & 6 & 3,2 & 0 & 0,0 & 0 & 0,0 & 0 & 0,0 & 6 & 3,2 \\
\hline 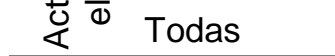 & 152 & 81,7 & 5 & 2,7 & 8 & 4,3 & 1 & 0,5 & 166 & 89,2 \\
\hline .02000 & 70 & 37,6 & 1 & 0,5 & 5 & 2,7 & 0 & 0,0 & 76 & 40,9 \\
\hline 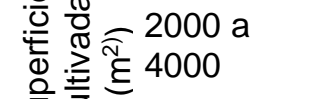 & 48 & 25,8 & 5 & 2,7 & 3 & 1,6 & 1 & 0,5 & 57 & 30,6 \\
\hline ळ ठ $>4000$ & 50 & 26,9 & 2 & 1,1 & 1 & 0,5 & 0 & 0,0 & 53 & 28,5 \\
\hline Frutas & 10 & 5,4 & 1 & 0,5 & 2 & 1,1 & 0 & 0,0 & 13 & 7,0 \\
\hline O & 99 & 53,2 & 4 & 2,2 & 4 & 2,2 & 0 & 0,0 & 107 & 57,5 \\
\hline $\begin{array}{ll}i \frac{2}{1} & \text { Frutas y } \\
& \text { hortalizas }\end{array}$ & 59 & 31,7 & 3 & 1,6 & 3 & 1,6 & 1 & 0,5 & 66 & 35,5 \\
\hline $\begin{array}{ll} & \text { Una vez } \\
\frac{0}{0}: & \text { por } \\
: \frac{0}{0}: & \text { semana }\end{array}$ & 48 & 25,8 & 2 & 1,1 & 1 & 0,5 & 1 & 0,5 & 52 & 28,0 \\
\hline 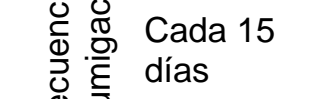 & 77 & 41,4 & 3 & 1,6 & 5 & 2,7 & 0 & 0,0 & 85 & 45,7 \\
\hline 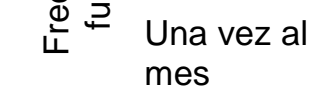 & 43 & 23,1 & 3 & 1,6 & 3 & 1,6 & 0 & 0,0 & 49 & 26,3 \\
\hline 드응 $<100$ & 20 & 10,8 & 2 & 1,1 & 2 & 1,1 & 1 & 0,5 & 26 & 14,0 \\
\hline ه 을 100 a 200 । & 120 & 64,5 & 5 & 2,7 & 5 & 2,7 & 0 & 0,0 & 130 & 69,9 \\
\hline $\bar{\infty} \frac{\pi}{0}>2001$ & 28 & 15,1 & 1 & 0,5 & 1 & 0,5 & 0 & 0,0 & 30 & 16,1 \\
\hline ฮ̊: & 42 & 22,6 & 2 & 1,1 & 4 & 2,2 & 0 & 0,0 & 48 & 25,8 \\
\hline $\begin{array}{l:l} & \frac{\bar{v}}{w} \bar{d} \text { a } 30 \\
\frac{0}{0} & \text { años }\end{array}$ & 87 & 46,8 & 4 & 2,2 & 3 & 1,6 & 1 & 0,5 & 95 & 51,1 \\
\hline 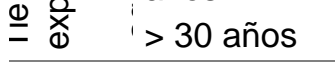 & 39 & 21,0 & 2 & 1,1 & 2 & 1,1 & 0 & 0,0 & 43 & 23,1 \\
\hline \multirow{2}{*}{ 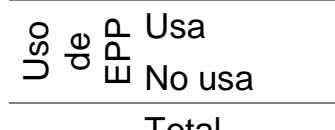 } & 63 & 33,9 & 4 & 2,2 & 3 & 1,6 & 0 & 0,0 & 70 & 37,6 \\
\hline & 105 & 56,5 & 4 & 2,2 & 6 & 3,2 & 1 & 0,5 & 116 & 62,4 \\
\hline Total & 168 & 90.3 & 8 & 4,3 & 9 & 4,8 & 1 & 0,5 & 186 & 100 \\
\hline
\end{tabular}

Nota: EPP: Equipo de protección personal. a: no se realiza la prueba Ji cuadrado por el número de casos muy bajo o cero en las celdas correspondientes a glóbulos rojos hipocrómicos normocíticos. 
Uso de organofosforados por agricultores de la comunidad de Guaslán- Ecuador y los cambios hematológicos

En la Tabla 8, los cambios más representativos a nivel morfológico en los glóbulos blancos son los siguientes: neutrófilos con granulación tóxica que aparecen como respuesta a un cuadro infeccioso, inflamatorio o tóxico. •Neutrófilos con hipersegmentación son células que tiene más de 5 lobulaciones en el núcleo, se asocian entre otros con procesos infecciosos y linfocitos reactivos que aparecen en procesos virales.

Tabla 8. Morfología de glóbulos blancos y variables sociodemográficas, actividades de los agricultores y de fumigación

\begin{tabular}{|c|c|c|c|c|c|c|c|c|c|c|c|c|}
\hline \multicolumn{2}{|c|}{$\begin{array}{l}\text { Morfología células } \\
\text { sanguíneas serie blanca }\end{array}$} & \multicolumn{2}{|c|}{$\begin{array}{l}\text { Neutró } \\
\text { filos } \\
\text { con } \\
\text { granul } \\
\text { ación } \\
\text { tóxica }\end{array}$} & \multicolumn{2}{|c|}{$\begin{array}{l}\text { Linfocitos } \\
\text { reactivos }\end{array}$} & \multicolumn{2}{|c|}{$\begin{array}{c}\text { Neutrófilos } \\
\text { con } \\
\text { granulació } \\
\text { n tóxica y } \\
\text { Lo } \\
\text { reactivos }\end{array}$} & \multicolumn{2}{|c|}{$\begin{array}{c}\text { Sin } \\
\text { alternacione } \\
\qquad\end{array}$} & \multicolumn{2}{|c|}{$\begin{array}{l}\text { Total en } \\
\text { muestra }\end{array}$} & \multirow[t]{2}{*}{ p } \\
\hline Variable & Categoría & $\mathrm{n}$ & $\%$ & $\mathbf{n}$ & $\%$ & $\mathbf{n}$ & $\%$ & $\mathbf{n}$ & $\%$ & $\mathbf{n}$ & $\%$ & \\
\hline \multirow{2}{*}{ 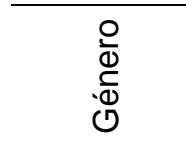 } & Hombre & 4 & 2,2 & 10 & 5,4 & 50 & 26,9 & 24 & 12,9 & 88 & 47,3 & \multirow{2}{*}{0,237} \\
\hline & Mujer & 10 & 5,4 & 17 & 9,1 & 51 & 27,4 & 20 & 10,8 & 98 & 52,7 & \\
\hline \multirow{3}{*}{$\begin{array}{l}\overline{0} \\
\frac{\pi}{0} \\
\text { ய }\end{array}$} & 18 a 28 años & 4 & 2,2 & 5 & 2,7 & 23 & 12,4 & 11 & 5,9 & 43 & 23,1 & \multirow{3}{*}{0,736} \\
\hline & 29 a 49 años & 9 & 4,8 & 16 & 8,6 & 50 & 26,9 & 23 & 12,4 & 98 & 52,7 & \\
\hline & > 49 años & 1 & 0,5 & 6 & 3,2 & 28 & 15,1 & 10 & 5,4 & 45 & 24,2 & \\
\hline \multirow{4}{*}{ 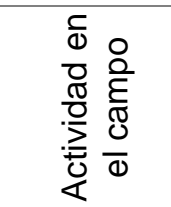 } & Fumiga & 0 & 0,0 & 1 & 0,5 & 5 & 2,7 & 3 & 1,6 & 9 & 4,8 & \multirow{4}{*}{ a } \\
\hline & Siembra & 0 & 0,0 & 3 & 1,6 & 1 & 0,5 & 1 & 0,5 & 5 & 2,7 & \\
\hline & Cosecha & 1 & 0,5 & 2 & 1,1 & 1 & 0,5 & 2 & 1,1 & 6 & 3,2 & \\
\hline & Todas & 13 & 7,0 & 21 & 11,3 & 94 & 50,5 & 38 & 20,4 & 166 & 89,2 & \\
\hline \multirow{3}{*}{ 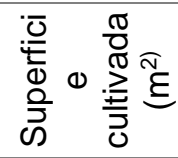 } & $<2000$ & 9 & 4,8 & 10 & 5,4 & 40 & 21,5 & 17 & 9,1 & 76 & 40,9 & \multirow{3}{*}{0,261} \\
\hline & 2000 a 4000 & 5 & 2,7 & 9 & 4,8 & 32 & 17,2 & 11 & 5,9 & 57 & 30,6 & \\
\hline & $>4000$ & 0 & 0,0 & 8 & 4,3 & 29 & 15,6 & 16 & 8,6 & 53 & 28,5 & \\
\hline \multirow{3}{*}{ 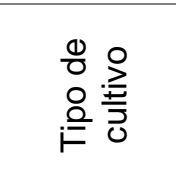 } & Frutas & 0 & 0,0 & 4 & 2,2 & 6 & 3,2 & 3 & 1,6 & 13 & 7,0 & \multirow{3}{*}{ a } \\
\hline & Hortalizas & 12 & 6,5 & 12 & 6,5 & 58 & 31,2 & 25 & 13,4 & 107 & 57,5 & \\
\hline & $\begin{array}{l}\text { Frutas y } \\
\text { hortalizas }\end{array}$ & 2 & 1,1 & 11 & 5,9 & 37 & 19,9 & 16 & 8,6 & 66 & 35,5 & \\
\hline \multirow{3}{*}{ 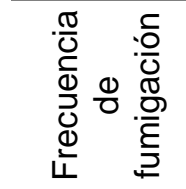 } & $\begin{array}{l}\text { Una vez por } \\
\text { semana }\end{array}$ & 2 & 1,1 & 8 & 4,3 & 27 & 14,5 & 15 & 8,1 & 52 & 28,0 & \multirow{3}{*}{0,376} \\
\hline & Cada 15 días & 6 & 3,2 & 9 & 4,8 & 51 & 27,4 & 19 & 10,2 & 85 & 45,7 & \\
\hline & Una vez al mes & 6 & 3,2 & 10 & 5,4 & 23 & 12,4 & 10 & 5,4 & 49 & 26,3 & \\
\hline \multirow{3}{*}{ 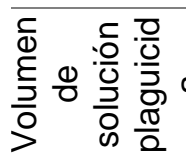 } & $<100 \mid$ & 1 & 0,5 & 5 & 2,7 & 10 & 5,4 & 10 & 5,4 & 26 & 14,0 & \multirow{3}{*}{ a } \\
\hline & 100 a 200 I & 12 & 6,5 & 16 & 8,6 & 76 & 40,9 & 26 & 14,0 & 130 & 69,9 & \\
\hline & $>2001$ & 1 & 0,5 & 6 & 3,2 & 15 & 8,1 & 8 & 4,3 & 30 & 16,1 & \\
\hline \multirow{3}{*}{ 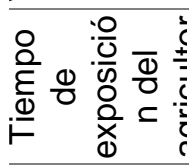 } & $<15$ años & 4 & 2,2 & 7 & 3,8 & 22 & 11,8 & 15 & 8,1 & 48 & 25,8 & \multirow{3}{*}{0,388} \\
\hline & 15 a 30 años & 8 & 4,3 & 17 & 9,1 & 51 & 27,4 & 19 & 10,2 & 95 & 51,1 & \\
\hline & $>30$ años & 2 & 1,1 & 3 & 1,6 & 28 & 15,1 & 10 & 5,4 & 43 & 23,1 & \\
\hline \multirow{3}{*}{ 욕욤 } & Usa & 4 & 2,2 & 10 & 5,4 & 40 & 21,5 & 16 & 8,6 & 70 & 37,6 & \multirow{2}{*}{0,875} \\
\hline & No usa & 10 & 5,4 & 17 & 9,1 & 61 & 32,8 & 28 & 15,1 & 116 & 62,4 & \\
\hline & Total & & & & & & & & & 186 & 100 & \\
\hline
\end{tabular}

Ciencia y Agricultura (Cien. Agri.) Vol. 17 (1). L-ISSN 0122-8420. elSSN 2539-0899.

Enero-Abril 2020, pp. 31-50. Tunja (Boyacá) - Colombia. DOI: https://doi.org/10.19053/01228420.v17.n1.2020.10603 
Nota: EPP: Equipo de protección personal. a: no se realiza la prueba Ji cuadrado por el número de casos muy bajo o cero en las celdas.

Del total de la muestra estudiada el $28 \%$ de los agricultores presentan plaquetas grandes mientras y el $72 \%$ presentan plaquetas con morfología normal.

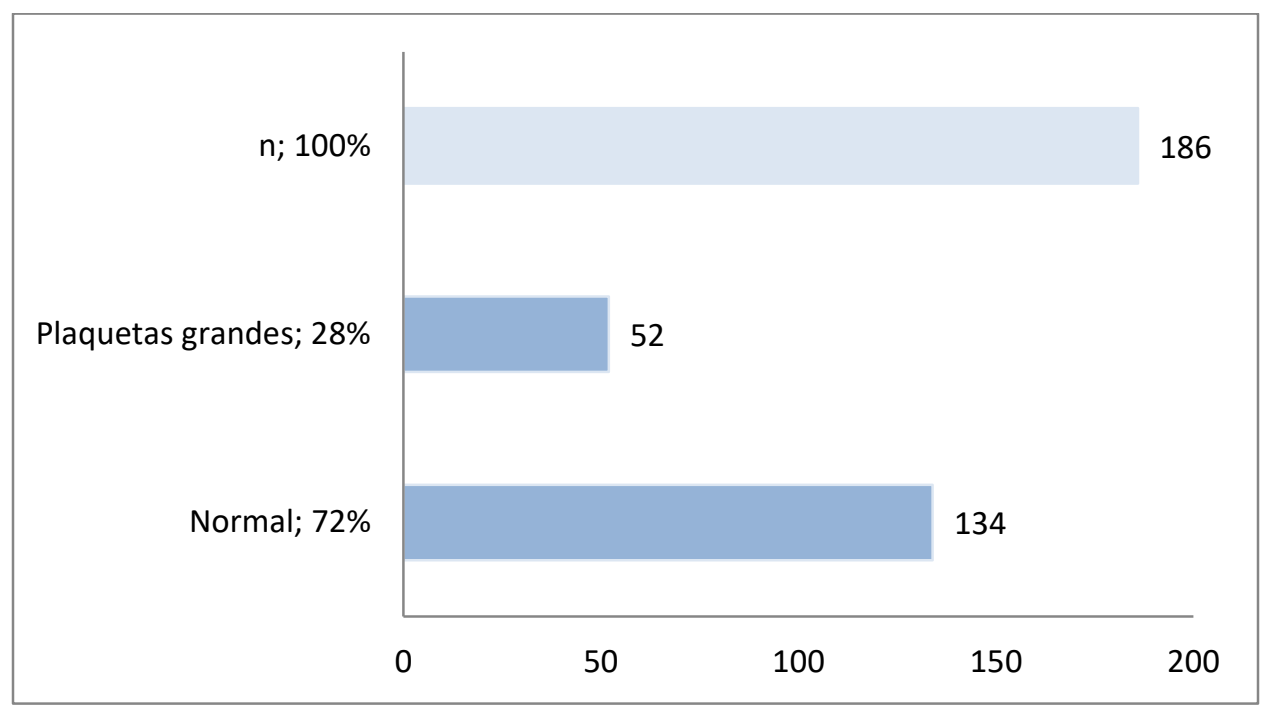

Fig. 3. Porcentaje de plaquetas con morfología alterada y normal.

En la Tabla 9 se evidencian cambios en $14,5 \%$ de plaquetas grandes en agricultores entre 29 - 49 años de edad y el 10,8\% de ellos poseen menos de $2000 \mathrm{~m}^{2}$ de tierras cultivadas, el $17,2 \%$ de agricultores presentó plaquetas grandes al fumigar cada 15 días, utilizan de 100 a 200 litros de solución (21\%) y han mantenido contacto con estos agentes químicos durante 25 a 30 años(15,3\%) por último los agricultores que no utilizan equipo de protección individual 18,3\% y al microscopio óptico refleja la presencia de plaquetas grandes.

La asociación estadísticamente significativa es para la relación entre la morfología de las plaquetas y la frecuencia de fumigación para un $p$ de 0,025.

Tabla 9. Morfología de plaquetas y variables sociodemográficas, actividades de los agricultores y de fumigación.

\begin{tabular}{|c|c|c|c|c|c|c|c|c|}
\hline \multicolumn{2}{|c|}{ Morfología de plaquetas } & \multicolumn{2}{|c|}{$\begin{array}{c}\text { Sin } \\
\text { alteraciones }\end{array}$} & \multicolumn{2}{|c|}{$\begin{array}{l}\text { Plaquetas } \\
\text { grandes }\end{array}$} & \multicolumn{2}{|c|}{$\begin{array}{l}\text { Total en } \\
\text { muestra }\end{array}$} & \multirow[t]{2}{*}{$\mathbf{p}$} \\
\hline Variable & Categoría & $\mathbf{n}$ & $\%$ & n & $\%$ & $\mathbf{n}$ & $\%$ & \\
\hline \multirow{2}{*}{ 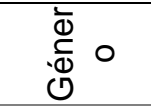 } & Hombre & 65 & 34,9 & 23 & 12,4 & 88 & 47,3 & \multirow{2}{*}{0,156} \\
\hline & Mujer & 69 & 37,1 & 29 & 15,6 & 98 & 52,7 & \\
\hline \multirow{3}{*}{ 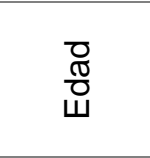 } & 18 a 28 años & 30 & 16,1 & 13 & 7,0 & 43 & 23,1 & \multirow{3}{*}{0,925} \\
\hline & 29 a 49 años & 71 & 38,2 & 27 & 14,5 & 98 & 52,7 & \\
\hline & $>49$ años & 33 & 17,7 & 12 & 6,5 & 45 & 24,2 & \\
\hline \multirow{4}{*}{ 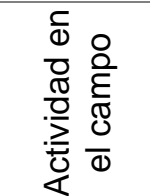 } & Fumiga & 8 & 4,3 & 1 & 0,5 & 9 & 4,8 & \multirow{4}{*}{0,631} \\
\hline & Siembra & 3 & 1,6 & 2 & 1,1 & 5 & 2,7 & \\
\hline & Cosecha & 4 & 2,2 & 2 & 1,1 & 6 & 3,2 & \\
\hline & Todas & 119 & 64,0 & 47 & 25,3 & 166 & 89,2 & \\
\hline
\end{tabular}


Uso de organofosforados por agricultores de la comunidad de Guaslán- Ecuador y los cambios hematológicos

\begin{tabular}{|c|c|c|c|c|c|c|c|c|}
\hline \multicolumn{2}{|c|}{ Morfología de plaquetas } & \multicolumn{2}{|c|}{$\begin{array}{c}\text { Sin } \\
\text { alteraciones }\end{array}$} & \multicolumn{2}{|c|}{$\begin{array}{c}\text { Plaquetas } \\
\text { grandes }\end{array}$} & \multicolumn{2}{|c|}{$\begin{array}{l}\text { Total en } \\
\text { muestra }\end{array}$} & \multirow{2}{*}{$\mathbf{p}$} \\
\hline Variable & Categoría & $\mathbf{n}$ & $\%$ & n & $\%$ & $\mathbf{n}$ & $\%$ & \\
\hline \multirow{3}{*}{ 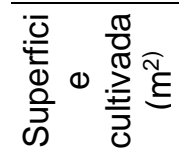 } & $<2000$ & 56 & 30,1 & 20 & 10,8 & 76 & 40,9 & \multirow{3}{*}{0,765} \\
\hline & 2000 a 4000 & 39 & 21,0 & 18 & 9,7 & 57 & 30,6 & \\
\hline & $>4000$ & 39 & 21,0 & 14 & 7,5 & 53 & 28,5 & \\
\hline \multirow{3}{*}{ 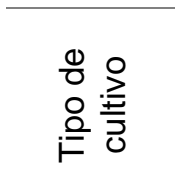 } & Frutas & 7 & 3,8 & 6 & 3,2 & 13 & 7,0 & \multirow{3}{*}{0,311} \\
\hline & Hortalizas & 78 & 41,9 & 29 & 15,6 & 107 & 57,5 & \\
\hline & Frutas y hortalizas & 49 & 26,3 & 17 & 9,1 & 66 & 35,5 & \\
\hline \multirow{3}{*}{ 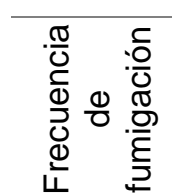 } & $\begin{array}{l}\text { Una vez por } \\
\text { semana }\end{array}$ & 41 & 22,0 & 11 & 5,9 & 52 & 28,0 & \multirow{3}{*}{$0,025^{\star}$} \\
\hline & $\begin{array}{l}\text { semana } \\
\text { Cada } 15 \text { días }\end{array}$ & 53 & 28,5 & 32 & 17,2 & 85 & 45,7 & \\
\hline & Una vez al mes & 40 & 21,5 & 9 & 4,8 & 49 & 26,3 & \\
\hline \multirow{3}{*}{ 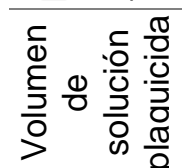 } & $<100$ & 21 & 11,3 & 5 & 2,7 & 26 & 14,0 & \multirow{3}{*}{0,528} \\
\hline & 100 a 200 I & 91 & 48,9 & 39 & 21,0 & 130 & 69,9 & \\
\hline & $>2001$ & 22 & 11,8 & 8 & 4,3 & 30 & 16,1 & \\
\hline \multirow{3}{*}{ 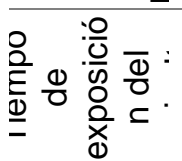 } & $<15$ años & 35 & 18,8 & 13 & 7,0 & 48 & 25,8 & \multirow{3}{*}{0,884} \\
\hline & 15 a 30 años & 67 & 36,0 & 28 & 15,1 & 95 & 51,1 & \\
\hline & > 30 años & 32 & 17,2 & 11 & 5,9 & 43 & 23,1 & \\
\hline \multirow{3}{*}{ 윰 $\frac{0}{0}$} & Usa & 52 & 28,0 & 18 & 9,7 & 70 & 37,6 & \multirow{2}{*}{0,597} \\
\hline & No usa & 82 & 44,1 & 34 & 18,3 & 116 & 62,4 & \\
\hline & Total & 134 & 70.0 & 52 & 30.0 & 186 & 100 & \\
\hline
\end{tabular}

Nota: p: Prueba Ji cuadrado, * asociación estadísticamente significativa; EPP: Equipo de protección personal.

En la Figura 4 la presencia de colinesterasa disminuida en un $13,4 \%$ de los agricultores indica posibles problemas de hemotoxicidad en los agricultores del sector de Guaslán, parroquia de San Luis.

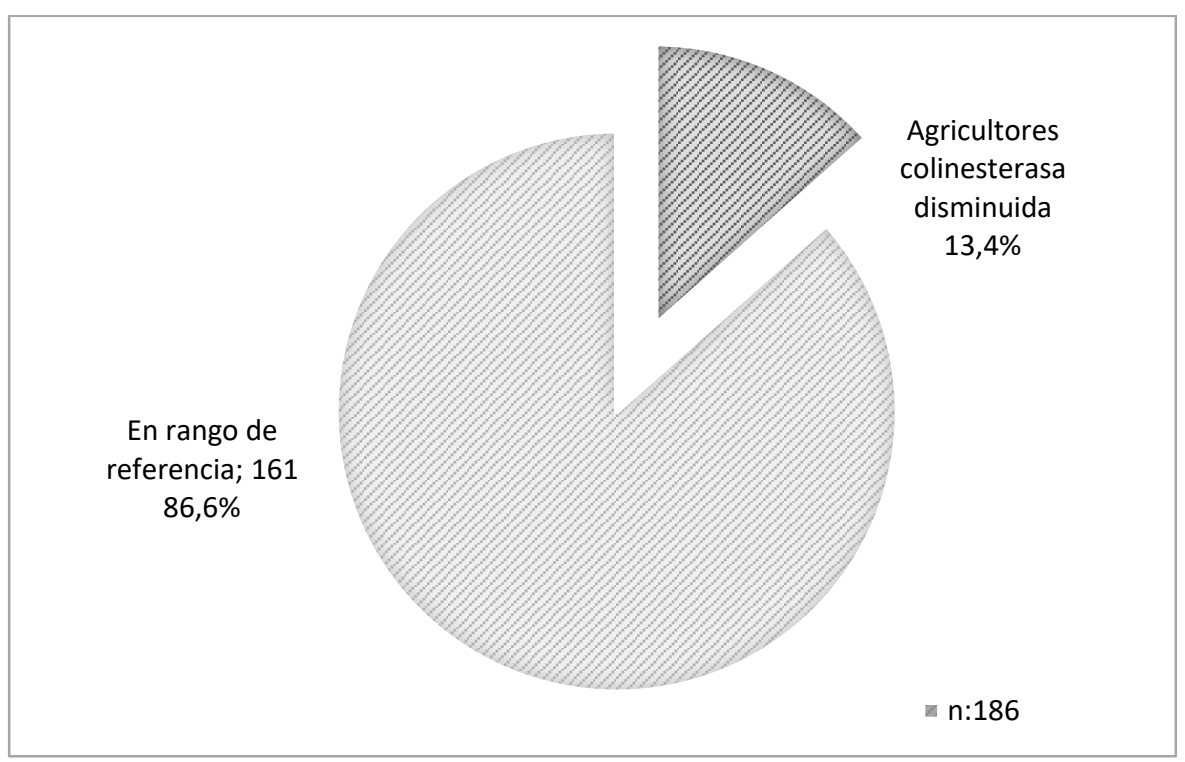

Fig. 4. Niveles de concentración de colinesterasa eritrocitaria.

Ciencia y Agricultura (Cien. Agri.) Vol. 17 (1). L-ISSN 0122-8420. eISSN 2539-0899. Enero-Abril 2020, pp. 31-50. Tunja (Boyacá) - Colombia. DOI: https://doi.org/10.19053/01228420.v17.n1.2020.10603 


\section{Discusıón}

Los agricultores que están expuestos ocupacionalmente a plaguicidas organofosforados en el país tienen una alta frecuencia en uso y manipulación de estos agroquímicos, lo que genera un problema de salud pública por ser aplicados en varias zonas agrícolas a nivel nacional, lo que se corrobora con lo dicho por Naranjo A, 2017 de Acción Ecológica, que indica que los agrotóxicos pasaron de ser una amenaza a ser un riesgo de salud pública.

En la población que se estudió, el $99 \%$ de las personas obtuvo más de una alteración en los parámetros evaluados en la biometría hemática. Los parámetros de hemoglobina $(\mathrm{Hb})$ y hematocrito (Hct) se encontraron significativamente elevados entre $65 \%$ y $52 \%$ de la población estudiada; un estudio realizado en Colombia demostró que la $\mathrm{Hb}$ y Hct se encontraron aumentados en $6 \%$ y $12 \%$ de un grupo de trabajadores de empresas privadas que estaban en contacto con plaguicidas organofosforados (Cortés, 2017). 111 agricultores presentaron contajes altos de glóbulos rojos,(parámetro hematológico) con un porcentaje del $60 \%$ de la población con valores fuera del rango de referencia, solamente el $2 \%$ de la población tuvo hemoglobina corpuscular media (HCM) baja y $82 \%$ presentaron concentración de hemoglobina corpuscular media ( $\mathrm{CHCM}$ ) disminuido, coincide este porcentaje con un estudio realizado en la India en donde los valores encontrados bajos los límites se observó en Hct, Hb, MCV, MCHC comparados con campesinos agricultores que usaban agrotóxicos y que fueron relacionados con problemas respiratorios descritos por Fareed et al. (2013).en contraparte con las normas establecidas en ese país para la fabricación de pesticidas donde crecen las expectativas de ser un potencial productor, con el apoyo de tecnología extranjera.

Del grupo de agricultores, 121 (65,1\%) obtuvo valores de hemoglobina sobre los valores de referencia, de los cuales $68(36,6 \%)$ fueron hombres y $53(28,5 \%)$ mujeres. El hematocrito se elevó en 32 agricultores $(17,2 \%)$ hombres y $65(35,9 \%)$ mujeres, con un total de 97 (52,1\%).

La poliglobulia encontrada podría ser valorada como un signo de adaptación fisiológica en personas que viven en las alturas, según lo dicho por Gallagher (2004), que coinciden con las variaciones encontradas en Colombia con poblaciones eminentemente agrícolas, lo que lleva a pensar que se deberían realizar mayores investigaciones en población agrícola a bajas alturas.

El recuento de glóbulos blancos presentó una elevación del $10 \%$ de la población (18 personas) lo que coincide con los datos descritos en el estudio de Cortés (2017), el cual -menciona que el $12 \%$ del grupo estudiado presentó leucocitosis, al igual que en los estudios realizados por Wafa et al. (2013) y Hundekari et al. (2013)(Hundekari, Suryakar, \& Rathi, 2013)este último autor corrobora también con el estudio donde el $29 \%$ de agricultores presentó neutrofilia, que sugiere que el desarrollo de leucocitosis puede deberse a un mecanismo de defensa del 
Uso de organofosforados por agricultores de la comunidad de Guaslán- Ecuador y los cambios hematológicos

sistema inmunológico frente a la intoxicación por organofosforados. Por otro lado, la población linfoide se valoró fuera del rango de referencia en un $34 \%$, de los cuales el 30\% reportó linfocitosis (con presencia de algunos linfocitos reactivos), al igual que los resultados obtenidos por Wafa et al. (2013) y Cortés (2017), en sus investigaciones.

De 186 agricultores, solo cinco personas presentaron contajes de plaquetas fuera de los rangos de referencia, tres tuvieron trombocitosis y dos trombocitopenias. Las plaquetas elevadas están relacionadas con los hallazgos obtenidos en el estudio de García et al. (2016)(García-García et al., 2016) y Wafa et al. (2013), así como el estudio Fareed et al. (2013), indicado como un mecanismo de defensa. Figueiredo et al. (2011)(De Figueiredo, Trape, \& Alonzo, 2011) y Díaz et al. (2001)(Diaz V, Pistilli N, Guillén N, Melgarejo MV, 2001), concuerdan al referirse al contaje de plaquetas disminuidas, valores menores a $150\left(10^{3} / \mathrm{mm}^{3}\right)$ El $20 \%$ de pacientes con plaquetas bajas (trombocitopenia de tipo inmune) se relaciona con enfermedades de tipo crónico por lo que es muy importante la realización de frotis sanguíneo EBMT (Group, 2011).

El frotis sanguíneo reflejó cambios en los glóbulos rojos como la estomatocitosis lo que hace referencia a la lipoperoxidación que se presenta en estados de intoxicación con organofosforados entre otros y que permite el aumento del estrés oxidativo en las células interactuando perjudicialmente con las membranas de las células, las proteínas y los ácidos nucleicos en general. Además, en el frotis se observaron glóbulos blancos con granulación tóxica, vacuolización y segmentados hiperlobulados (hipersegmentados) todos indicativos de presencia de un proceso infeccioso generalmente de tipo agudo. Según lo indicado por Campuzano (2008), el análisis del frotis de sangre es uno de los parámetros más informativos que el profesional de laboratorio puede realizar y ningún equipo automatizado hasta la fecha puede suplantarlo, por esa razón se reitera la importancia de realizar el frotis sanguíneo como estudio en los cambios que se pueden presentar en personas intoxicadas con pesticidas en este caso con organofosforados.

Mediante la determinación de la colinesterasa eritrocitaria se estableció que la actividad enzimática de este analito estaba disminuida y $13 \%$ de agricultores presentaron este valor $(n=25)$, coincidiendo con los datos obtenidos por Hongsibsong et al. (2017), Ibarra (2012) y Cobano (2018) en sus estudios.

La población con rango de edad entre 29 a 49 años que realizaban todas las actividades en el campo, en una superficie menor a $2000 \mathrm{~m}^{2}$, que sembraban hortalizas, utilizaban una cantidad de plaguicida entre 100 a 200 litros, con periodos de fumigación de una vez cada 15 días, con un tiempo de exposición de 25 a 30 años y que no utilizaban equipos de protección personal (EPP) fueron los más afectados en cuanto a las alteraciones celulares cualitativas y cuantitativas.

Es importante resaltar que el estudio de Figueiredo et al. (2010),el $79 \%$ de la población estudiada no utilizó implementos de protección para la fumigación, de igual manera Hongsibsong (2017) indicó que no existe implementos 
estandarizados de protección o usan simples pañuelos que tapan nariz y boca que no protegen lo suficiente al agricultor, lo que concuerda con esta investigación, se observaron agricultores que no ocupaban adecuadamente sus ropa de protección a pesar de existir instituciones que entregan implementos de fumigación no son utilizados evidenciando falta de capacitación y responsabilidad en los agricultores que no los utilizan. Deben existir normas que exijan el cumplimiento para el manejo de estos pesticidas resaltando la necesidad por parte de autoridades en salud que disminuiría el riesgo en salud y mantendría una mejor calidad de vida en los agricultores que tienen tan complicada tarea en el campo.

\section{AgRADECIMIENTOS}

Al Gobierno Autónomo Descentralizado del San Luis y su presidente el Sr. Leonardo Santillán y a la comunidad de Guaslán-Ecuador que han permitido que sea posible realizar este estudio.

\section{Referencias}

Campuzano, G. (2008). Utilidad del extendido de sangre periférica: los leucocitos*. Colombia. Recuperado de: https://www.medigraphic.com/pdfs/medlab/myl-2008/myl089-10b.pdf

Cobano, I. (2018). Indicadores de efecto en la prevención de intoxicaciones agudas por organofosforados y carbamatos. Universidad de Sevilla. Recuperado de: https://idus.us.es/xmlui/handle/11441/82626

Cortez, S. C. (2017). Evaluación de los parámetros hematológicos: Cuadro hemático y frotis de sangre periférica, en trabajadores expuestos a pesticidas organofosforados, carbamatos y piretroides. Cundinamarca 2016-2017. Bogotá D. C.: Universidad Nacional de Colombia. Recuperado de: http://bdigital.unal.edu.co/58052/1/53068721.2017.pdf

De Figueiredo, G. M., Trape, A. Z., \& Alonzo, H. A. (2011). Exposição a múltiplos agrotóxicos e prováveis efeitos a longo prazo à saúde: Estudo transversal em amostra de 370 trabalhadores rurais de Campinas (SP). Revista Brasileira de Medicina Do Trabalho, 9(1), 1-9.

Diaz, V., Pistilli, N., Guillén, N., \& Melgarejo, M.V. (2001). Valores Hematologicos en individuos expuestos accidentalmente a insecticidas organofosforados. Mem. Inst. Investig. Cienc. Salud, 1(1), 1-4.

Fernández, D., Mancipe, L., \& Fernández, D. (2010). Intoxicación por organofosforados. SEMERGEN Medicina de Familia, 33 (1), 21-23. https://doi.org/10.1016/S1138-3593(07)73839-X

Gallagher, S. A., \& Hackett, P. H. (2004). High-altitude illness. Emergency Medicine Clinics of North America, 22(2), 329-355. https://doi.org/10.1016/i.emc.2004.02.001

García-García, C. R., Parrón, T., Requena, M., Alarcón, R., Tsatsakis, A. M., \& Hernández, A. F. (2016). Occupational pesticide exposure and adverse health effects at the clinical, hematological and biochemical level. Life Sciences, 145, 274-283. https://doi.org/10.1016/j.lfs.2015.10.013

Group EBMT (2011). Trombocitopenia inmune. Recuperado de: https://www.ebmt.org/sites/default/files/migration_legacy_files/document/EBMT_Practical_Guides_for_N urses_Immune_Thrombocytopenia_Spanish.pdf

Hongsibsong, S., Sittitoon, N., \& Sapbamrer, R. (2017). Association of health symptoms with low-level exposure to organophosphates, DNA damage, AChE activity, and occupational knowledge and practice among rice, corn, and double-crop farmers. Journal of Occupational Health, 59(2), 165-176. https://doi.org/10.1539/joh.16-0107-OA

Hundekari, I. A., Suryakar, A. N., \& Rathi, D. B. (2013). Acute organo-phosphorus pesticide poisoning in North Karnataka, India: Oxidative damage, haemoglobin level and total leukocyte. African Health Sciences, 13(1), 129-136. https://doi.org/10.4314/ahs.v13i1.18

Ibarra, E., \& Linares, T. (2012). Colinesterasa sérica como biomarcador de exposición a compuestos organofosforados y carbamatos. Revisión crítica, 13(537), 59-65.

INEC (2013). Modulo Ambiental: Uso de Plaguicidas en la Agricultura, 1-15.

INEC (2016). Ecuador en cifras/compendio estadístico | Instituto Nacional de Estadística y Censos.

Mora Ortiz, L. E. (2014). Manejo de plaguicidas e impacto en la salud de los trabajadores que cultivan cebolla, Jubones, Cantón Santa Isabel, 2014. Universidad de Cuenca. Recuperado de: http://dspace.ucuenca.edu.ec/bitstream/123456789/23671/1/TESIS.pdf

Naranjo, A.-A. (2017). La otra guerra: la situación de los plaguicidas en el Ecuador. Quito. Recuperado de: http://www.swissaid.org.ec/sites/default/files/images/plaguicidas web.pdf

Sáenz, F. K., Narváez, G, L., \& Cruz, M. (2008). Revista Mexicana de Patologia Clinica. Revista

Ciencia y Agricultura (Cien. Agri.) Vol. 17 (1). L-ISSN 0122-8420. elSSN 2539-0899.

Enero-Abril 2020, pp. 31-50. Tunja (Boyacá) - Colombia. DOI:

https://doi.org/10.19053/01228420.v17.n1.2020.10603 
Uso de organofosforados por agricultores de la comunidad de Guaslán- Ecuador y los cambios hematológicos

Latinoamericana de Patología Clínica y Medicina de Laboratorio, 55(4), 207-215.

Santillán, L., Morocho, E., \& Aucancela, L. S. (2014). Gobierno Autónomo Descentralizado Parroquial Rural San Luis, periodo 2014-2019. Recuperado de: http://app.sni.gob.ec/snilink/sni/portal sni/data sigad plus/sigadplusdiagnostico/0660819820001 diagnostico pdyot 2015 san luis 30-10-2015 13-28-45.pdf

Toro, B., Rojas, A., \& Díaz, J. (2017). Niveles de colinesterasa sérica en caficultores del Departamento de Caldas, Colombia. Rev. Salud Pública, 19(3), 318-324. https://doi.org/10.15446/rsap.v19n3.52742

Wafa, T., Nadia, K., Amel, N., Ikbal, C., Insaf, T., Asma, K., \& Mohamed, H. (2013). Oxidative stress, hematological and biochemical alterations in farmers exposed to pesticides. Journal of Environmental Science and Health - Part B Pesticides, Food Contaminants, and Agricultural Wastes, 48(12), 10581069. https://doi.org/10.1080/03601234.2013.824285

Ciencia y Agricultura (Cien. Agri.) Vol. 17 (1). L-ISSN 0122-8420. elSSN 2539-0899.

Enero-Abril 2020, pp. 31-50. Tunja (Boyacá) - Colombia. DOI:

https://doi.org/10.19053/01228420.v17.n1.2020.10603 